an Axin-LRP complex through increased phosphorylation and avidity involving the presence of the multiple Axin-binding motifs (Fig. 2).

The tightly bound Axin-LRP complex may be the site at which $\beta$-catenin turnover is extinguished, perhaps through processes involving endocytosis or titration of GSK-3 from Axin ${ }^{18-20}$. In this model, the binding of Axin to LRP would initiate an irreversible process of ever-increasing phosphorylation leading to the recruitment of the endocytic machinery or the titration of GSK-3 from Axin by Frizzled receptor-associated $G$ proteins ${ }^{20}$. This process could be initiated by a threshold level of LRP phosphorylation. In this context, the identification of CK1 $\gamma$ as an essential but unregulated component may reflect its role in establishing a threshold of CK1 activity at the membrane. So is there any evidence for a Dishevelledassociated kinase whose activity is regulated by Wnt ligands? Again, a CK1 isoform steps forward. Swiatek et al. have shown that CK1E activity is upregulated by Wnt ligands after

\section{Spying on the inner life of a cell}

Life isn't simple. And neither is gene activity. dephosphorylation of its autoinhibitory C terminus ${ }^{21}$. The enhancement of LRP phosphorylation by Dishevelled-associated kinases may also explain the role of Dishevelled in recruiting Axin to the plasma membrane during signaling 22 .

The further analysis of early Wnt signaling will be a difficult undertaking because of the dynamic positive and negative roles of key components. A useful analogy may be to regard the $\beta$-catenin turnover complex as a molecular machine in which kinases such as GSK-3 and CK1 function as cogs in several processes. The trick required to understand the machine's function may be the removal of selected cogs at defined times. This will require a fine set of molecular tools.

\footnotetext{
1. Davidson, G. et al. Nature 438, 867-872 (2005).

2. Zeng, X. et al. Nature 438, 873-877 (2005).

3. Schwarz-Romond, T. et al. Genes Dev. 16, 2073 2084 (2002).

4. Logan, C.Y. \& Nusse, R. Annu. Rev. Cell Dev. Biol. 20, 781-810 (2004).

5. Ding, Y. \& Dale, T. Trends Biochem. Sci. 27, 327-
} 329 (2002)
6. Knippschild, U. et al. Cell. Signal. 17, 675-689 (2005).

7. Liu, C. et al. Cell 108, 837-847 (2002)

8. Amit, S. et al. Genes Dev. 16, 1066-1076 (2002).

9. Yanagawa, S. et al. EMBO J. 21, 1733-1742 (2002).

10. Cong, F., Schweizer, L. \& Varmus, H. Mol. Cell. Biol. 24, 2000-2011 (2004).

11. Tamai, K. et al. Mol. Cell 13, 149-156 (2004).

12. He, X., Semenov, M., Tamai, K. \& Zeng, X. Development 131, 1663-1677 (2004).

13. Marin, O. et al. Proc. Natl. Acad. Sci. USA 100 , 10193-10200 (2003).

14. Mao, J. et al. Mol. Cell 7, 801-809 (2001).

15. Wong, H.C. et al. Mol. Cell 12, 1251-1260 (2003).

16. Tamai, K. et al. Nature 407, 530-535 (2000).

17. Cong, F. \& Varmus, H. Development 131, 51035108 (2004).

18. Chen, W. et al. Science 301, 1391-1394 (2003).

19. Castellone, M.D., Teramoto, H., Williams, B.O. Druey, K.M. \& Gutkind, J.S. Science 310, 1504 1510 (2005).

20. Liu, X., Rubin, J.S. \& Kimmel, A.R. Curr. Biol. 15 1989-1997 (2005).

21. Swiatek, W. et al. J. Biol. Chem. 279, 13011-13017 (2004).

22. Cliffe, A., Hamada, F. \& Bienz, M. Curr. Biol. 13 960-966 (2003).
The stages involved in expressing a protein are numerous, and because each step is governed by probability, the levels of mRNA and protein within a cell are subject to great variability. Yet the cell must perform certain critical functions and respond appropriately to external stimuli, despite fluctuation in the levels of factors essential for the response. Consequently, studies of the kinetics of gene expression at the singlecell level are necessary to help elucidate how cells function in a noisy environment.

Studies of gene expression have suggested a strong distinction between prokaryotes and eukaryotes with regard to its regulation. Data from bacteria suggest that a gene expresses relatively small numbers of mRNAs and that translation occurs in bursts. In eukaryotes, which have the added complexity of a highly organized chromatin structure, the ability to open chromatin for bursts of transcription seems to be more important in terms of overall expression.

Previous studies were able to measure protein levels precisely, but mRNA levels could only be estimated. What was needed was a way in which the limiting factor, mRNA, could be directly measured, and this is accomplished in a new study by Golding et al. (Cell 123, 1025-1036, 2005). The EscHerichia coli system they developed has two components: an RNA-binding protein, MS2, fused to GFP and the RFP gene fused to 96 tandem copies of the MS2-binding site. Thus, with appropriate normalization, the focal green signal can be directly related to the level of mRNA, whereas the diffuse red signal corresponds to the protein level. As shown in the figure, each cell shows different levels of both mRNA and protein that can be quantified.

The first conclusion of these studies was that, in fact, expression in bacteria at the single-cell level is more similar to previous eukaryotic data: mRNA expression occurs in intense bursts, which

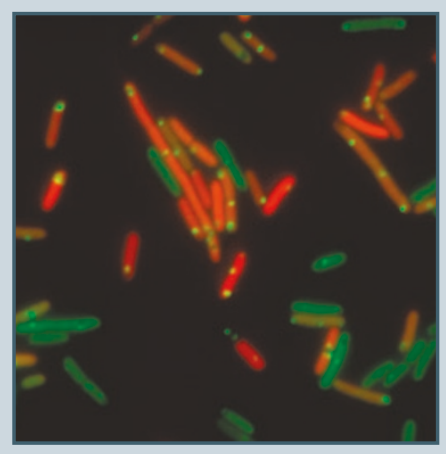
synthesize a random number of transcripts. This is an approximately Poisson process (that is, each burst of transcription produces a geometric distribution of mRNAs). What happens to the mRNAs when the cell divides? Here the data fit exactly to a binomial distribution, providing direct proof that partitioning is random, as expected.

The correlation between transcript and protein levels was determined. It has been assumed that there would be proportionality between the two, and this is observed in older cells. In newly divided cells, however, the correlation is significantly weaker. Golding et al. propose that this results from the randomization of mRNAs during division. Protein levels, being high, are distributed virtually equally in the two daughter cells, but transcripts, being fewer in number, are less likely to segregate equally; this magnifies differences in the two cells. As the cell grows, however, this effect is neutralized by protein expression. On average, each mRNA produces $60-110$ protein molecules.

This work on the kinetics of gene expression in single cells provides novel insight into a stochastic process at a resolution that was previously unattainable. The results indicate that our views about chromatin remodeling as a rate-limiting step in gene expression may need to be reconsidered, and further experiments are required to understand the nature of the burst process.

Angela K Eggleston 